\title{
3-D Imaging using Row-Column-Addressed 2-D Arrays with a Diverging Lens: Phantom Study
}

Bouzari, Hamed; Engholm, Mathias; Beers, Christopher; Stuart, Matthias Bo; Nikolov, Svetoslav Ivanov; Thomsen, Erik Vilain; Jensen, Jørgen Arendt

Published in:

2017 IEEE International Ultrasonics Symposium (IUS)

Link to article, DOI:

10.1109/ULTSYM.2017.8092496

Publication date:

2017

Document Version

Peer reviewed version

Link back to DTU Orbit

Citation (APA):

Bouzari, H., Engholm, M., Beers, C., Stuart, M. B., Nikolov, S. I., Thomsen, E. V., \& Jensen, J. A. (2017). 3-D Imaging using Row-Column-Addressed 2-D Arrays with a Diverging Lens: Phantom Study. In 2017 IEEE International Ultrasonics Symposium (IUS) IEEE. https://doi.org/10.1109/ULTSYM.2017.8092496

\section{General rights}

Copyright and moral rights for the publications made accessible in the public portal are retained by the authors and/or other copyright owners and it is a condition of accessing publications that users recognise and abide by the legal requirements associated with these rights.

- Users may download and print one copy of any publication from the public portal for the purpose of private study or research.

- You may not further distribute the material or use it for any profit-making activity or commercial gain

- You may freely distribute the URL identifying the publication in the public portal 


\title{
3-D Imaging using Row-Column-Addressed 2-D Arrays with a Diverging Lens: Phantom Study
}

\author{
Hamed Bouzari*, Mathias Engholm ${ }^{\dagger}$, Christopher Beers ${ }^{\ddagger}$, Matthias Bo Stuart*, \\ Svetoslav Ivanov Nikolov ${ }^{\S}$, Erik Vilain Thomsen ${ }^{\dagger}$, and Jørgen Arendt Jensen* \\ * Dept. of Elec. Eng., Bldg. 349, Technical University of Denmark, 2800 Kgs. Lyngby, Denmark \\ $\dagger$ Dept. of Micro- and Nanotechnology, Technical University of Denmark, 2800 Kgs. Lyngby, Denmark \\ $¥$ Sound Technology Inc, Analogic Ultrasound Group, PA-16803, USA \\ $\S$ BK Ultrasound ApS, 2730 Herlev, Denmark
}

\begin{abstract}
A double-curved diverging lens over a flat rowcolumn-addressed (RCA) 2-D array can extend its inherent rectilinear 3-D imaging field-of-view (FOV) to a curvilinear volume region, which is necessary for applications such as abdominal and cardiac imaging. A concave lens with radius of $12.7 \mathrm{~mm}$ was manufactured using RTV664 silicone. The diverging properties of the lens were evaluated based on measurements on several phantoms. The measured $6 \mathrm{~dB}$ FOV in contact with a material similar to human soft tissue was less than $15 \%$ different from the theoretical predictions, i.e., a curvilinear FOV of $32^{\circ} \times 32^{\circ}$. A synthetic aperture imaging sequence with single element transmissions was designed for imaging down to $14 \mathrm{~cm}$ at a volume rate of $88 \mathrm{~Hz}$. The performance was evaluated in terms of signal-to-noise ratio (SNR), FOV, and full-widthat-half-maximum (FWHM). The penetration depth in a tissue mimicking phantom with $0.5 \mathrm{~dB} /(\mathrm{cm} \mathrm{MHz})$ attenuation was $13 \mathrm{~cm}$. The results of this study confirm that the proposed lens approach is an effective method for increasing the FOV, when imaging with RCA 2-D arrays.
\end{abstract}

\section{INTRODUCTION}

An $N \times N$ element 2-D array can be operated utilizing only $2 N$ connections, when a row-column, cross-electrode, or toporthogonal-to-bottom-electrode (TOBE) addressing scheme is used [1]-[4]. This is contrary to the $N^{2}$ connections needed, when fully addressing the elements. In general, a row-columnaddressed (RCA) array is a 2-D matrix array, which is addressed via its row and column indices. Effectively, it consists of two 1-D arrays arranged orthogonal to each other.

It has been demonstrated in several studies that row-column technology is a realistic alternative to the state-of-the-art matrix probes, especially as a low-cost alternative. However, two major issues with the RCA arrays is that they can only emit acoustic energy directly in front of the array in a cross-shape to the sides, and only one-way focusing is possible in each lateral direction. Therefore the contrast and spatial resolutions are compromised compared with fully-addressed arrays of the same physical size. The contrast and spatial resolutions can be compensated for by using an apodization scheme, as well as increasing the size of the array [5]. However, imaging can only be performed in a rectilinear region in front of the array. For cardiac imaging, it is relevant to have a probe with a small foot-print capable of phased array imaging, such that the heart can be visualized through the ribs. True volumetric phased array imaging is possible with RCA arrays, provided that the array is double curved to spread the energy during transmit [6]. In [7] it was shown how to make a curved transducer element by bending the capacitive micromachined ultrasonic transducer (CMUT) RCA array in one dimension. However, manufacturing double curved transducer elements in two dimensions is challenging for both CMUT and piezoelectric transducer technologies. Another approach to spread the acoustic energy uses a double curved diverging acoustic lens on top of the RCA array [8], [9]. Using a lens makes it easier to fabricate curved arrays, as it is not needed to manufacture curved elements, and also making a lens is a well-tested technology. An in-depth study of the possibilities in this approach has been previously investigated based on simulations by the authors [9].

In this paper, a more detailed discussion on the pros and cons of using a diverging lens based on measurements on phantoms is presented. The curvilinear volumetric imaging performance of an RCA array equipped with a mountable diverging lens is investigated in terms of field-of-view (FOV), spatial and contrast resolution, as well as the SNR measurements using synthetic aperture imaging (SAI) technique.

The paper is organized as follows: In the following Section II, the lens parameters are explained. Section III presents the utilized SAI sequence, a detailed overview of the measurements setup, as well as the quality assessment measures. Section IV explains and discusses the measurement results. The final Section concludes the paper.

\section{Double-Curved Diverging Acoustic Lens}

Using a double-curved RCA 2-D array extends the volumetric imaging FOV of a flat RCA 2-D array to a curvilinear region. To spread the acoustic energy of a line-element curvilinearly along its larger dimension, it has to be curved like an arc to generate a diverging wave. Another approach is to use a doublecurved diverging acoustic lens on top of the flat RCA array. The red dashed lines are illustrating the wave fronts at different time instances. A beamforming approach was proposed in [9] to accurately calculate the correct time-of-flights of the wave fronts using a diverging lens, and hence avoiding geometrical distortions. However, for the proposed beamfoming approach, the curvature of the wave fronts has to be estimated based on the physical properties of the lens in contact with the imaging medium. In this section a lens model adapted from optics 
into acoustics will be described. To simplify this model, the reflection from the boundaries inside the lens as well as the attenuation effects are neglected.

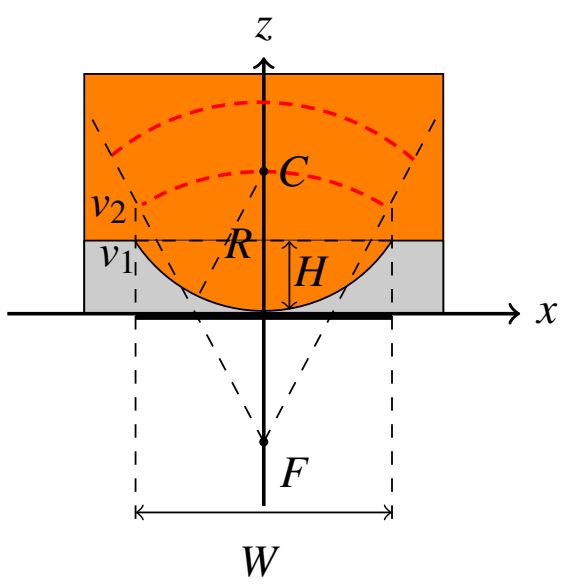

Fig. 1. A diverging lens with a material that has a constant speed of sound, $v_{1}$, can be manufactured in concave shape. The orange colored material in (a), $v_{2}$, has the same properties as human soft tissue.

For a thin concave lens, where the radius is much larger than the arc height, the focal distance can be estimated from (1), which is the first-order Taylor expansion of the total surface power, i.e., $P=1 / F$, of the lens at its both flat and spherical surfaces [10]:

$$
\frac{1}{F} \approx\left(1-\frac{v_{1}}{v_{2}}\right) \frac{1}{R},
$$

where $v_{1}$ and $v_{2}$ are the speed of sound in the lens material and the medium. Depending on the application, the medium can be water, human soft tissue, or any other material. The focal length of the lens is $F$ and the radius of the arc is $R$. To have a larger curvature, i.e., $1 / R$, the ratio $v_{1} / v_{2}$ has to increase, and the curvature of the lens and the diverged wave fronts are exactly the same, when $v_{1} / v_{2}=2$. A positive value of the focal length indicates that the lens is converging the wave fronts, and a negative value indicates that it is diverging.

The f-number, $f_{\#}$, and FOV of the lens can be defined as:

$$
\begin{gathered}
f_{\#}=\frac{F}{W}, \\
\mathrm{FOV}=2 \operatorname{arccot}\left(2 f_{\#}\right) .
\end{gathered}
$$

The theoretical $f_{\#}$ is between $0<f_{\#}<\infty$, which corresponds to $\pi>$ FOV $>0$. A FOV of zero means no divergence is occurring and only the rectilinear region in front of the array can be imaged, similar to flat RCA 2-D arrays. In practice, the lens chord, $W$ and its thickness, $H$ are both limited by the arrays aperture size as well as the attenuation through the lens, therefore to increase the FOV, the ratio $v_{1} / v_{2}$ has to increase. However, it can be practically difficult to find materials with large velocity ratio and low impedance ratio, therefore, lower $f_{\#}$ values are less feasible.
Table I

TRANSDUCER AND THE LENS PARAMETERS AND SETUP CONFIGURATION

\begin{tabular}{llc} 
Center frequency & 3 & $\mathrm{MHz}$ \\
Pitch row & 270 & $\mu \mathrm{m}$ \\
Pitch column & 270 & $\mu \mathrm{m}$ \\
Number of rows (columns) & 62 & - \\
Pulse repetition frequency & 5 & $\mathrm{kHz}$ \\
No. of active elements in Tx & 1 & - \\
Scan depth (max range) & 14 & $\mathrm{~cm}$ \\
Sinusoid emission cycles & 2 & - \\
Focus in receive & Dynamic & - \\
Synthetic Tx apodization & Hann. & - \\
Rx electronic apodization & Hann. & - \\
Sampling frequency & 70 & $\mathrm{MHz}$ \\
Tx voltage & \pm 75 & $\mathrm{~V}$ \\
Lens $f_{\#}$ & -1.5 & - \\
Sound speed in $20^{\circ} \mathrm{C}$ water & 1482 & $\mathrm{~m} / \mathrm{s}$ \\
Sound speed in vivo & 1540 & $\mathrm{~m} / \mathrm{s}$ \\
RTV664 silicone sound speed & 1000 & $\mathrm{~m} / \mathrm{s}$ \\
RTV664 longitudinal atten. (at $3 \mathrm{MHz})$ & 1.4 & $\mathrm{~dB} / \mathrm{mm}$ \\
\hline
\end{tabular}

\section{METHODS}

A SAI sequence is designed for imaging down to $14 \mathrm{~cm}$ of depth. It utilizes single element transmissions on the row elements, and the echoes are collected with all the column elements. For a speed of sound of $1540 \mathrm{~m} / \mathrm{s}, 182 \mu \mathrm{s}$ is required to acquire a single image line to a depth of $14 \mathrm{~cm}$ including penetrating the lens. For 62 emissions this is equivalent to a volume rate of $88 \mathrm{~Hz}$, and it is the same sequence used in the simulation study in [9]. Hilbert transformed RF data are used for beamforming a low-resolution volume for every emission and finally, by summing all the low-resolution volumes in phase, a high-resolution volume is generated.

The transducer parameters of a PZT RCA $62+62$ element 2-D array as well as the imaging setup configuration are shown in Table I. The probe is connected to the experimental ultrasound scanner SARUS [11]. The measured Hilbert transformed RF signals are beamformed using a MATLAB (MathWorks Inc., Massachusetts, USA) implementation of the delay-andsum (DAS) beamformer specific to curved RCA arrays [9]. To remove the otherwise apparent secondary echoes originating from the either ends of line-elements, roll-off apodization regions are placed at both ends of each element [3], [12]. The length of each apodization region was equal to 15 times the pitch of the array. Theoretically, transmitting with row elements and receiving with column elements should image exactly the same curvilinear volume as transmitting with column elements and receiving with row elements. Thus, no preference is considered in transmitting with row elements and receiving the echoes with column elements, or vice versa.

The add-on lens was made by casting room temperature vulcanization (RTV) silicone, RTV664 (Momentive Performance 


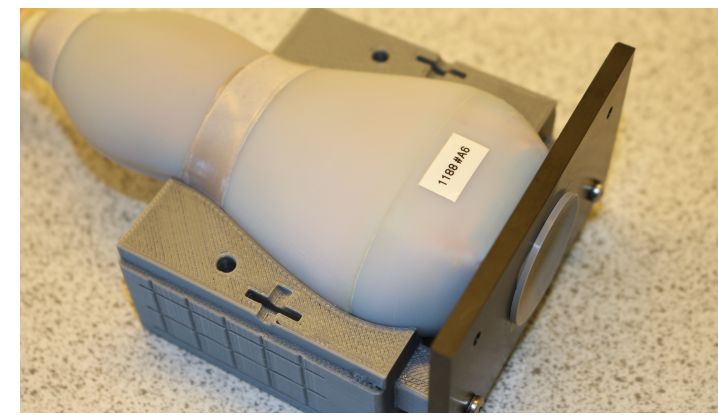

Fig. 2. The lens modules are placed in front of the probe using a holder (half of the holder is shown in the figure).

Materials Inc., New York, USA ), into a rigid plastic frame and using a stainless steel ball bearing to form the curved surface. A mold assembly was made, consisting of a flat bottom plate and a top plate with a circular hole in which a steel ball sat during curing. The frame was sandwiched between these two plates. During cure, pressure was applied to push the ball down into the mold assembly.

Fig. ?? illustrates the schematics of the concave lens with a radius of $12.7 \mathrm{~mm}$ made out of RTV664 silicone, which has a lower speed of sound $(1000 \mathrm{~m} / \mathrm{s})$ compared to human soft tissue $(1540 \mathrm{~m} / \mathrm{s})$ and therefore follows the design shown in Fig. II. The defocusing aperture as well as the height of the spherical cap for the $12.7 \mathrm{~mm}$ radius lens are $23.56 \mathrm{~mm}$ and $7.96 \mathrm{~mm}$ The minimum thickness at the center of the lens is $0.75 \mathrm{~mm}$. The longitudinal attenuation coefficient in the RTV664 silicone is approximately $1.4 \mathrm{~dB} / \mathrm{mm}$ at $3 \mathrm{MHz}$. That corresponds to an axial attenuation of $12.19 \mathrm{~dB}$ for the $12.7 \mathrm{~mm}$ radius lens at its largest thickness at the corners. The concave cavity of the lens is filled with ultrasound gel.

To evaluate the imaging performance of the lens, several ultrasound phantoms are used. A geometrical copper wire phantom was used as line targets, where wires were located at different depths with $1 \mathrm{~cm}$ spacing. The wire grid phantom has eleven columns separated by $1 \mathrm{~cm}$ and each has 12 rows of wires. A tissue mimicking phantom with cylindrical anechoic targets, model 571 from Danish Phantom Design (Frederikssund, Denmark) with an attenuation of $0.5 \mathrm{~dB} /(\mathrm{cm} \mathrm{MHz})$ was used for signal-to-noise ratio (SNR) and contrast measurements.

The transmit pressure measurements of the lens was carried out using an AIMS III intensity measurement system (Onda Corporation, Sunnyvale, California, USA) connected to the experimental research scanner SARUS [13], [14]. The OptiSon ultrasound beam analyzer (Onda Corporation, Sunnyvale, California, USA) was used to validate the diverging properties of the lens in a water tank. The current prototyped probes do not have the required safety permissions to be used on humans, therefore no in vivo data have been acquired.

\section{RESUlts AND Discussion}

Using (1), the focal length of the lens with radius $12.7 \mathrm{~mm}$ in contact with water is $-39.04 \mathrm{~mm}$. For human soft tissue, the focal length is $-36.21 \mathrm{~mm}$. It can be noticed that the curvature

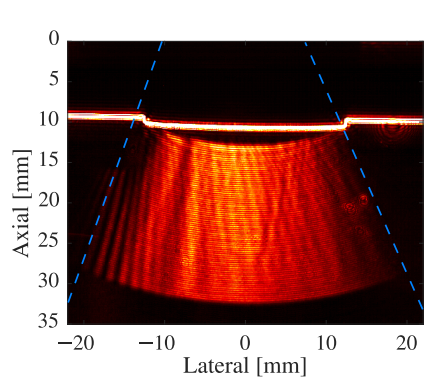

(a)

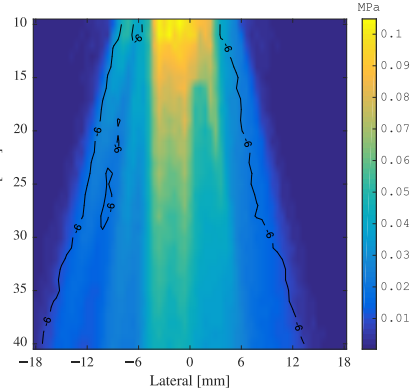

(b)
Fig. 3. Simulated and measured transmit acoustic field: (a) OptiSon ultrasound beam analyzer (Onda Corporation, Sunnyvale, CA, USA), and (b) measured with hydrophone (Onda Corporation, Sunnyvale, CA, USA) in a water tank. The origin corresponds to the center of the lens.

of the wave fronts are smaller in water compared with human tissue. Based on the estimated focal lengths, the effect of the lens can be represented as a virtual arc shaped elements with the appropriate curvatures for the beamforming [9].

To validate the diverging properties of the lens, Fig. 3a shows the optical projection of the density gradient generated by the acoustic pressure in water based on the Schlieren imaging concept. The data were measured using the OptiSon ultrasound beam analyzer (Onda Corporation, Sunnyvale, CA, USA). A burst of sinusoidal excitation pulses at $3 \mathrm{MHz}$ center frequency was transmitted using one element near the center of the array. The lens was not centered accurately on the probe during the measurement, as observed by the slight asymmetry of the beam profiles in Fig. 3a. The measured FOV from Fig. 3a is $38.42^{\circ}$. The measured FOV is larger compared with the theoretical estimation, which is due to the higher dynamic range of the optical images in Fig. 3a, i.e., 40-dB. The visible FOV boundaries is indicated by blue dashed lines in Fig. 3a.

Fig. $3 b$ shows the transmit pressure beam in a water tank. The data was measured using a hydrophone (Onda Corporation, Sunnyvale, CA, USA) in a lateral plane. At each measurement location, the maximum negative pressure was recorded. The measured FOV for the $6 \mathrm{~dB}$ contour plots is $33.39^{\circ}$. The measured FOV in contact with water is smaller than the theoretical estimation in contact with human soft tissue, due to their different speeds of sound in (1).

Fig. 4 illustrates three cross-planes (azimuth, elevation, and C-plane) of a wire grid phantom as well as an anechoic cyst phantom imaged with the lens. In the elevation plane in Fig. $4 \mathrm{e}$, the whole of the straight wires are not visible, that is because the reflections from the wires travel away from the transducer at the either ends of the wire.

A volume region of a tissue mimicking phantom with $0.5 \mathrm{~dB} /(\mathrm{cm} \mathrm{MHz})$ attenuation and no cysts was imaged 20 times for calculating the SNR. Using the lens with radius $12.7 \mathrm{~mm}$ has a penetration depth of around $13 \mathrm{~cm}$ for single element transmissions. To minimize the reflection and the attenuation through the lens, a compounded lens made out of two or more different materials can be made, but this is beyond the scope 


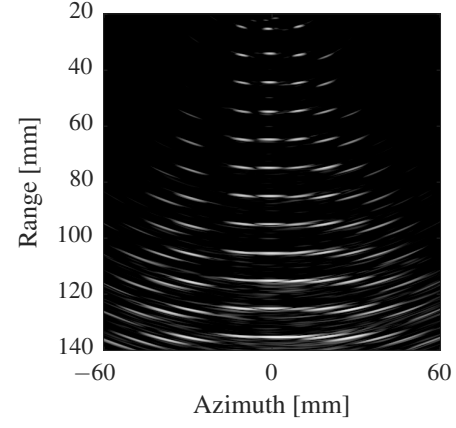

(a)

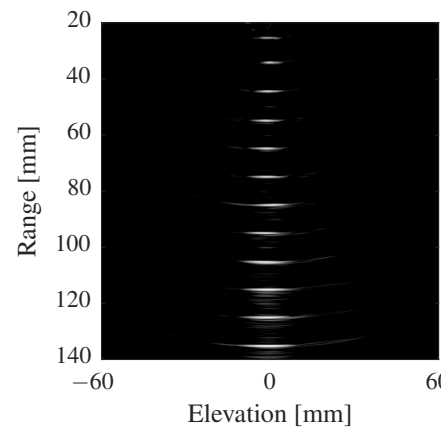

(c)

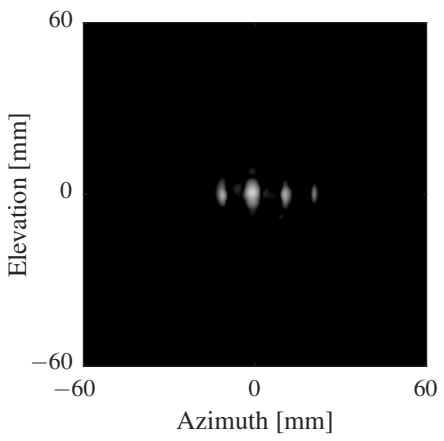

(e)

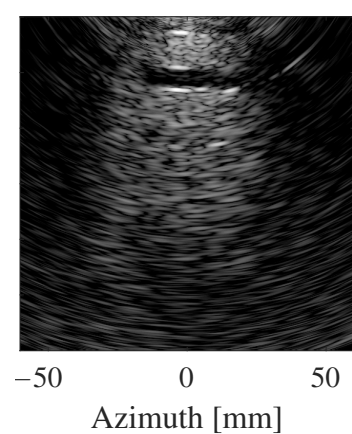

(b)

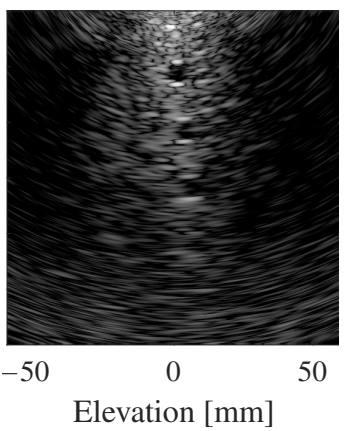

(d)

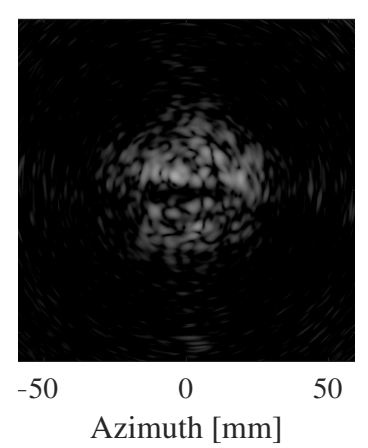

(f)
Fig. 4. Three cross planes (azimuth, elevation, and C-plane) of hollow cyst and wire grid phantoms imaged with lens are shown in a 40-dB dynamic range, (left column) for the $25.4 \mathrm{~mm}$ radius lens and (right column) for the $12.7 \mathrm{~mm}$ radius lens. The $\mathrm{C}$-planes are at a depth of $42 \mathrm{~mm}$. (a) and (b) Azimuth plane. (c) and (d) Elevation plane. (e) and (f) C-plane.

of this study.

\section{CONCLUSION}

In this paper the curvilinear imaging performance of a $62+62$ RCA 2-D PZT array was evaluated based on phantom studies using a mountable diverging lens. The lens had a $12.7 \mathrm{~mm}$ radius. Using a SAI sequence with single element emissions at a time, it was possible to image down to $14 \mathrm{~cm}$ at a volume rate of $88 \mathrm{~Hz}$. The capabilities of the lens to effectively diverge the acoustic beam was investigated using measurement with OptiSon beam analyzer as well as pressure measurement in water bath with a hydrophone. It was shown that the rectilinear imaging FOV of the flat RCA 2-D arrays can be increased to a curvilinear imaging FOV using diverging lens. In this study, the FOV was extended to $32^{\circ} \times 32^{\circ}$ in contact with a material having similar properties as human soft tissue. The measured FOV was less than $15 \%$ different from the theoretical predictions in contact with water, and it was less than $12 \%$ in contact with human soft tissue.

\section{ACKNOWLEDGMENT}

This work was financially supported by grant 82-2014-4 from the Danish National Advanced Technology Foundation and from BK Ultrasound ApS, Herlev, Denmark.

\section{REFERENCES}

[1] C. E. Morton and G. R. Lockwood, "Theoretical assessment of a crossed electrode 2-D array for 3-D imaging," in Proc. IEEE Ultrason. Symp., 2003, pp. 968-971.

[2] C. H. Seo and J. T. Yen, "A 256 x 256 2-D array transducer with rowcolumn addressing for 3-D rectilinear imaging," IEEE Trans. Ultrason., Ferroelec., Freq. Contr., vol. 56, no. 4, pp. 837-847, April 2009.

[3] M. F. Rasmussen, T. L. Christiansen, E. V. Thomsen, and J. A. Jensen, "3-D imaging using row-column-addressed arrays with integrated apodization - Part I: Apodization design and line element beamforming,' IEEE Trans. Ultrason., Ferroelec., Freq. Contr., vol. 62, no. 5, pp. 947958, 2015

[4] R. K. W. Chee, A. Sampaleanu, D. Rishi, and R. J. Zemp, "Top orthogonal to bottom electrode (TOBE) 2-D CMUT arrays for 3-D photoacoustic imaging," IEEE Trans. Ultrason., Ferroelec., Freq. Contr., vol. 61, no. 8, pp. 1393-1395, 2014.

[5] H. Bouzari, M. Engholm, C. Beers, S. I. Nikolov, M. B. Stuart, E. V. Thomsen, and J. A. Jensen, "CMUT and piezoelectric row-columnaddressed 2-D array probes - Part II: Imaging performance assessment on phantoms," IEEE Trans. Ultrason., Ferroelec., Freq. Contr., p. submitted, 2016.

[6] C. E. M. Démoré, A. Joyce, K. Wall, and G. Lockwood, "Real-time volume imaging using a crossed electrode array," IEEE Trans. Ultrason., Ferroelec., Freq. Contr., vol. 56, no. 6, pp. 1252-1261, 2009.

[7] A. I. H. Chen, L. L. P. Wong, S. Na, Z. Li, M. Macecek, and J. T. W. Yeow, "Fabrication of a curved row-column addressed capacitive micromachined ultrasonic transducer array," J. Microelectromech. S., vol. 25, no. 4, pp. 675-682, 2016.

[8] A. W. Joyce and G. R. Lockwood, "Crossed-array transducer for real-time 3D imaging," in Proc. IEEE Ultrason. Symp., 2014, pp. 2116-2120.

[9] H. Bouzari, M. Engholm, C. Beers, M. B. Stuart, S. I. Nikolov, E. V. Thomsen, and J. A. Jensen, "Curvilinear 3-D imaging using row-columnaddressed 2-D arrays with a diverging lens: Feasibility study," IEEE Trans. Ultrason., Ferroelec., Freq. Contr., vol. 64, no. 6, pp. 978-988, 2017.

[10] J. E. Greivenkamp, Field Guide to Geometrical Optics, ser. Field Guide Series. Society of Photo Optical, 2004.

[11] J. A. Jensen, H. Holten-Lund, R. T. Nilsson, M. Hansen, U. D. Larsen, R. P. Domsten, B. G. Tomov, M. B. Stuart, S. I. Nikolov, M. J. Pihl, Y. Du, J. H. Rasmussen, and M. F. Rasmussen, "SARUS: A synthetic aperture real-time ultrasound system," IEEE Trans. Ultrason., Ferroelec., Freq. Contr., vol. 60, no. 9, pp. 1838-1852, 2013.

[12] T. L. Christiansen, M. F. Rasmussen, J. P. Bagge, L. N. Moesner, J. A Jensen, and E. V. Thomsen, "3-D imaging using row-column-addressed arrays with integrated apodization - part II: Transducer fabrication and experimental results," IEEE Trans. Ultrason., Ferroelec., Freq. Contr., vol. 62, no. 5, pp. 959-971, 2015.

[13] J. A. Jensen, M. F. Rasmussen, M. J. Pihl, S. Holbek, C. A. VillagomezHoyos, D. P. Bradway, M. B. Stuart, and B. G. Tomov, "Safety assessment of advanced imaging sequences, I: Measurements," IEEE Trans. Ultrason., Ferroelec., Freq. Contr., vol. 63, no. 1, pp. 110-119, 2016.

[14] J. A. Jensen, "Safety assessment of advanced imaging sequences, II: Simulations," IEEE Trans. Ultrason., Ferroelec., Freq. Contr., vol. 63, no. 1, pp. 120-127, 2016. 